\title{
Cosmic Ray Detectors and Observational Breakthroughs in Atmospheric Electricity
}

\author{
Rasha Abbasi*广 \\ University of Utah \\ E-mail: rabbasi@luc.edu
}

\begin{abstract}
Recently, there have been several breakthroughs in the study of atmospheric electricity using ground based cosmic ray detectors. The observations reported from these cosmic ray detectors can lead to a better understanding of the physical mechanisms that initiate lightning. In addition, they allow the study of rare atmospheric phenomena such as, Terrestrial Gamma-ray Flashes (TGFs), and elves. In this talk, I will present some of the recent observations and their impact. I will also discuss the role of these cosmic ray detectors as the world's leading instruments for the detection of atmospheric electric fields, TGFs, and lightning initiation mechanisms. The successful use of ground based cosmic rays detectors, designed to study astrophysical phenomena, for the study of lighting in Earth's atmosphere attests to the vitality of this field of experimental research, which is now dedicated not only to solving the mysteries of the universe but also the mysteries of our own atmosphere.
\end{abstract}

36th International Cosmic Ray Conference -ICRC2019-

July 24th - August 1st, 2019

Madison, WI, U.S.A.

* Speaker.

${ }^{\dagger}$ A footnote may follow. 


\section{Introduction}

With the advancement of astroparticle detectors and experimental observations, we continue to better understand particle physics at energies unattainable by current particle accelerators. We also continue to further our understanding of energetic sources Kpcs-Mpcs away. On the other hand, thunderstorms occur only a few kilometers away from the Earth surface, and are of regular occurrence, and it may be assumed that they are well understood. The truth is, even though they have been a subject of intense research, basic questions about lightning initiation and propagation are still poorly understood.

Several cosmic ray experiments designed to detect high energy cosmic rays discovered their ability to study our atmosphere. As a result of such discoveries, there have recently been quite a few breakthroughs in our knowledge in atmospheric electricity. In fact, some of these cosmic ray experiments, are now the world's leading facilities in answering some of the top ten most important questions in atmospheric physics like: what is the origin of lightning? How does lightning propagate? What are the conditions that produce Terrestrial Gamma ray Flashes and Transient Luminous Events? [Dwyer and Uman, 2014].

The Telescope Array Surface Detector (TASD), the Grapes 3 Muon Telescope (G3MT), the Low-Frequency Array (LOFAR), and the Auger experiments are examples of detectors that have been contributing significantly to answering these question. In the following sections, I will describe in more detail each of these questions, the cosmic ray detectors and their observations. I will also discuss the importance of these observations in the current picture of our knowledge of atmospheric electric fields.

\section{Lightning initiation and propagation}

Thunderstorms are quite an energetic phenomenon of electric discharge. A major advance in the study of thunderclouds was achieved when their dipole structure was discovered [Rees, 1956]. One possible scenario for the charge separation in thunderclouds is that it occurs when graupel (light hail) and ice crystals come in grazing contact. The ice crystals turns more positive while the graupel becomes more negative. Upward wind drafts carry the lighter ice crystals higher up in the atmosphere to the top of the clouds (several kilometers away from the negatively charged graupel layer). Such charge separation produces an electric field. If the electric field is large enough to increase the energy of seed electrons (possibly from cosmic rays) so as to produce an avalanche from impact with air molecules that surpass the attachment rate, the number of electrons will grow exponentially with time and distance. This growth will produce low energy electron and ion discharges referred to as "leaders" (narrow channels in which large currents flow). Leaders propagate for tens of meter lengths, then pause then move again another step. Note that for leaders to occur, the electric field must reach a value of greater than $3 \times 10^{6} \mathrm{~V} / \mathrm{m} \times n_{\text {air }}$ (where $n_{\text {air }}$ is the density of air relative to that at sea level) for conventional breakdown to occur.

Knowledge of the electric field magnitude and structure inside the thunderstorm is key to understanding lightning initiation. The heart of the problem of understanding lightning initiation is that, with decades of electric fields measurements, the observed values of detected electric field are not sufficient to create a leader or a stroke propagating on a kilometer(s) scale [Stolzenburg et al., 2007a, 
Winn and other, 1974]. This could mean that either our understanding of how lightning is initiated, or that electric field measurements in thunderstorms, are inaccurate

Traditionally, balloons and planes are used to make such measurements. However, there are limitations to obtaining such electric field measurements. A Thunderstorm is a violent environment. Flying a balloon or an aircraft into the storm is difficult if not even dangerous. Moreover, the thunderstorm scale is large, and the fields inside thunderstorms change in a matter of seconds. Balloon or aircraft measurements only provide a small picture (localized measurements) of these fields. For the balloon or the aircraft to travel through the area inside the storm with the relevant electric field is of low probability.

Recently, scientists on LOFAR discovered their ability to provide measurements probing the atmospheric electric field in thunderstorms. Moreover, they realized their ability to use the LOFAR detector to map lightning in three dimensions in unprecedented detail. This allowed them to understand a more rare phenomenon in lightning leaders, referred to as positive leaders.

\subsection{Electric field magnitude and structure with LOFAR:}

There have been several balloon and aircraft experiments measuring the electric field inside a thunderstorm [Weber et al., 1982, Fitzgerald, 1984, Stolzenburg et al., 2007b]. However, these detectors can not fly throughout the whole structure of the thunderstorm, are effected by highly violent conditions, and may contribute to discharging the thunderstorm cloud with their presence.

Alternatively, detecting the electric field of a thunderstorm from the ground offers the opportunity to observe the thundercloud atmospheric electric system without perturbing it, while not being affected by violent weather conditions.

LOFAR offers such an opportunity with the detection of the cosmic ray extensive air shower (EAS) radio signal from the ground. LOFAR is the world's largest radio telescope observing astronomical sources and cosmic ray air showers [Winchen et al., 2019]. The LOFAR part used in this analysis is in the main core stations of LOFAR implemented in the Netherlands (at sea level). Each of these stations contain 96 low-band antennas (LBAs). The $\mathrm{V}$ antennas are implanted in pairs sensitive to orthogonal polarizations and detecting RF in the $10-90 \mathrm{MHz}$ range. The dominant noise in the LOFAR signal is the human radio frequency and the galactic background noise.

In fair weather days the observed radio signal of cosmic rays is expected to be polarized in a particular direction. This emission and polarization is a result of the relativistic electrons and positrons in the shower deflected by the Lorenz force due to the Earth's magnetic field. The polarization direction of the observed emission is predominantly in the $\hat{e}_{v \times B}$ direction with a minor radial deviation due to negative charge excess in the shower front. Here $V$ is the direction of the EAS propagation and $B$ is the Earth's magnetic field.

It was noted that in non-fair weather, where thunderstorms contain high electric fields, the polarization pattern of the observed cosmic ray events is expected and is observed to be different from those events observed under fair weather conditions (see Figure 1). In addition, the intensity patterns were found to be significantly different for both classes of events. The intensity pattern of the fair weather events normally shows a single maximum in the $\hat{e}_{v \times B}$, while thunderstorm events were found to follow a ring structure with a radius of about $100 \mathrm{~m}$ (see Figure 2).

Such a difference can be explained and modeled [Nelles et al., 2015] using CORSIKA, with FLUKA and QGSJETII. The radio emission simulation is generated by the CoREAS plug-in [Huege et al., 2013]. 
It was found that the dominent component of the electric field contributing to the observed LOFAR signal is the component perpendicular to the CR shower axis. The calculated electric field reproduces the main intensity and polarization pattern features observed in LOFAR radio data successfully [Schellart et al., 2015a]. The best fit is found for a proposed two layer model when assuming the upper layer resides between 2.9-8 km with an electric field magnitude of $E_{u}=50 \mathrm{kV} / \mathrm{m}$ and a lower layer resides between the ground and $2.9 \mathrm{~km}$ with a magnitude of $E_{L}=26.5 \mathrm{kV} / \mathrm{m}$ [Schellart et al., 2015b]. Note that $E_{L}$ is about half of the value of $E_{u}$ and points in the opposite direction.

Observing extensive air showers by their radio emission during thunderstorm conditions thus provides a unique way to probe the atmospheric electric fields in thunderclouds. LOFAR thus provides an opportunity to study the electric field inside the thunderstorms, without disturbing the electric field it's measuring, while also being sensitive to a large fraction of the cloud. This new technique may help answer the long standing question "how is lighting initiated in thunderclouds? “.
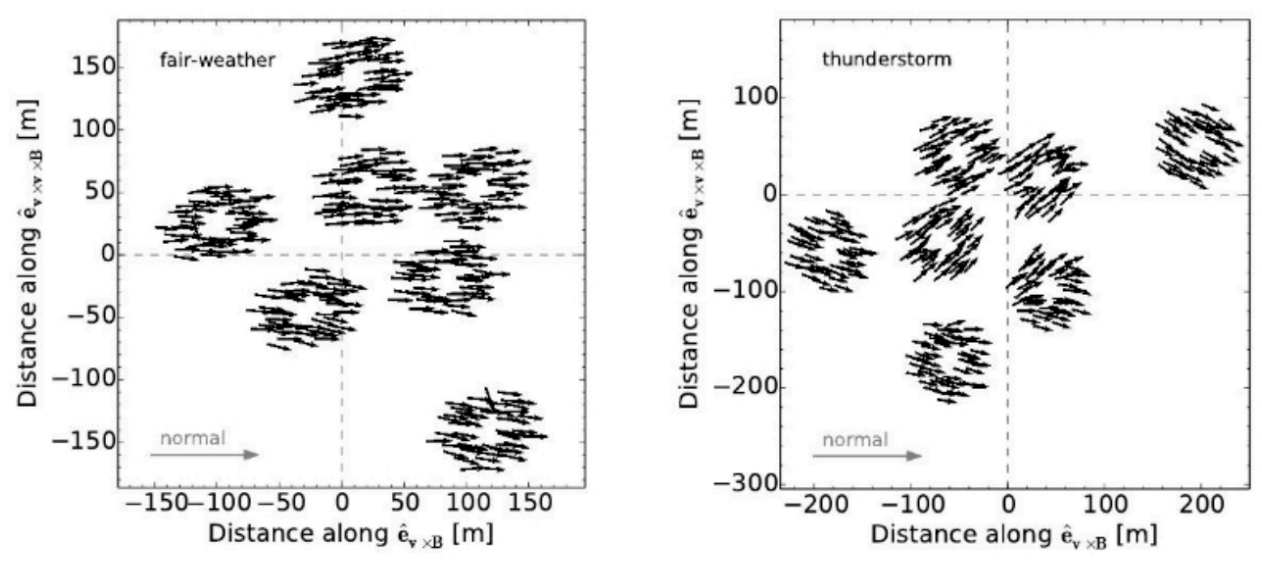

Figure 1: LOFAR antenna's are grouped into circular stations. Each antenna's polarization direction is shown with a black arrow. The y-axis represents the direction of $(v \times v \times B)$ and the $\mathrm{x}$-axis is the direction of $(v \times B)$. The left plot is the expected polarization direction for a "normal" fair-weather air shower. The right plot shows that the cosmic ray shower shows a different polarization than expected due to the thunderstorm.

\subsection{Mapping Lightning with LOFAR:}

To understand lightning initiation, characterization, and how lightning is related to gamma ray production in the atmosphere, the use of lightning mapping technology has been instrumental.

Most lightning mapping systems can be categorized using two main methods: Mapping lightning using 3D VHF Lightning Mapping Arrays(LMA) antennas and 2D Lightning Interferometer (INTF).

Lightning Mapping Arrays (LMAs) are normally composed of 6 to 20 VHF antennas, at frequencies between $60-80 \mathrm{MHz}$, located kilometers apart, and which could measure the time and the power of the lightning strikes [Rison et al., 1999a]. LMAs are ideal to detect isolated pulses that are emitted by breakdown process (i.e. stepped leader). 

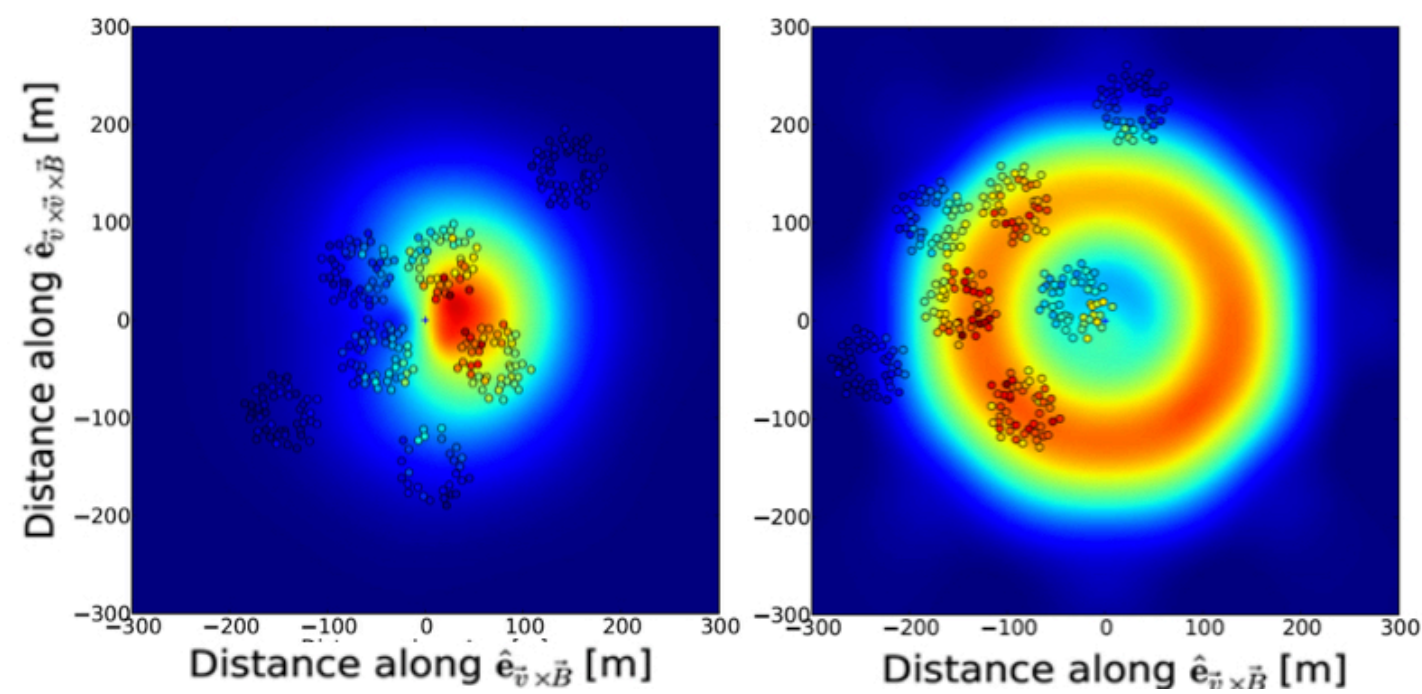

Figure 2: Radio intensity pattern for the cosmic ray event in fair-weather (left plot) and in a thunderstorm (right plot). The small circles represent the antenna positions while their color represents the measured pulse power. The color scale in the back represents the best-fitting simulation through COREAS. Note that the color of the circles matches that obtained by simulation. Also note that the intensity patterns are different for both events.

Typically, the INTF is composed of 3-4 antennas, at frequencies between 20-80 MHz, located meters apart. The INTF scans the lightning flash in 2D (azimuth and zenith angles) [Stock et al., 2014]. The INTF is ideal to study the quasi-continuous emissions from lightning pulses (i.e. dart leader). INTF also scans thousands more sources than the LMA [Rison et al., 1999b].

The LOFAR radio telescope scientists has recently developed a technique, making it the first system in the world that can detect lightning in 3D in potentially similar details to what the INTF provides in 2D [Hare et al., 2018]. This would allow us to probe the internal structure of leader propagation to a precision that was not available before.

Figure 3 shows the result mapping a lightning flash observed on On July 12th 2016, a lightning flash triggering 23 stations (12 core and 11 remote) by LOFAR stations. LOFAR uses the Time Of Arrival (TOA) technique [Hare et al., 2018] to locate as many lightning sources as possible. Each point in this map represent an event source. Panels A,B,C show the altitude of these sources vs. time, east-west, and temp. D and E show the NS-EW and NS-altitude.

A total of 1500 sources were located in $200 \mathrm{~ms}$ (about 7 sources per ms). To directly compare to a typical LMA/INTF event, a total of 1100 and 26000 sources were observed by LMA and INTF for a flash that lasted about $600 \mathrm{~ms}$ in 2004 [Stock et al., 2014].

Note that, with the LOFAR locating source algorithm under continuous improvement, they anticipate an improvement up to 50 sources per ms. This is comparable to INTF capabilities in 2D (about 100 source per ms). It is expected that the number of sources per ms should improve further for closer lightning events. With this capability, LOFAR is able for the first time to probe the internal structure of lightning leader.

One example of an important result from the detailed LOFAR mapping capability comes from studying the detailed internal structure of a positive stepped leaders. Positive stepped leaders in 
general are much less understood and observed in comparison to the better known negative stepped leaders. Positive leaders are defined as attracting free electrons while negative leaders repel free electrons. We know a lot less about positive leaders as opposed to negative leaders, since they do occur a lot less frequently, they are less luminous, and they emit less VHF signal.

Figure 4 shows a positive and a negative leader observed by LOFAR. From the LOFAR observations it was clear that positive and negative leaders propagate differently. The positive leaders' VHF signal is not detected from the main leader channel, as negative leaders are, but due to "needle" like shapes that are twinkling from that main channel [Hare, 2019].

Due to the unprecedented precision of LOFAR, scientists are able for the first time to study these "needle" like structures in-depth. The needle structure propagates for 30-100 m with a radius of less than $5 \mathrm{~m}$ and a speed of around $3 \times 10^{5} \mathrm{~m} / \mathrm{s}$, which is similar to the negative leader speed. This implies that the needles are a form of negative breakdown, moving charge away from the positive leader main channel. Several possible models are now attempting to explain this phenomenon on a much finer level than was possible before [Hare, 2019].

It is speculated that needle structures could explain the cause of multiple cloud-to-ground lightning events. This happens due to the fact that the main channel of lightning is not discharged all in a single flash, but is in part stored alongside the leader channel in the needles. The stored charge, then returns to the main channel, via needles and could in principle initiate further discharges [Hare, 2019].

LOFAR's pioneering efforts in mapping lightning, allows for the first time, a better understanding of the rarely occurring phenomena of positive leaders. This new and continuously improving mapping capability of lightning at LOFAR is an important tool for understanding lightning. 



Figure 3: Lightning mapped by LOFAR. Every point is a VHF source event. panel A shows altitude vs. time. panel B shows altitude vs. east-west. Panel C shows altitude vs. temperature. Panel D shows northsouth vs. east-west. Panel E shows north-south vs. altitude. The shade of the dots represent time dark is earlier light is later. 


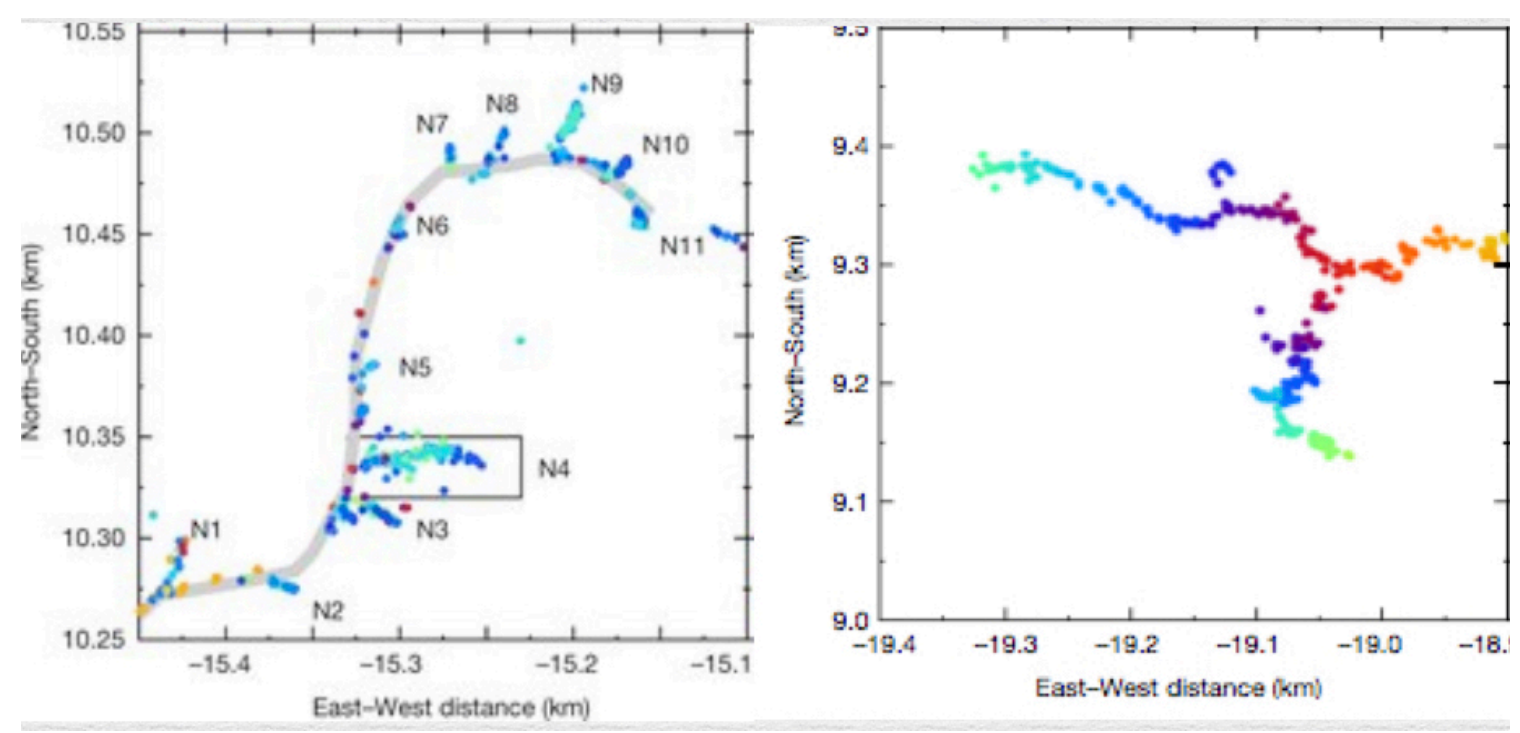

Figure 4: positive vs. negative leaders observations by LOFAR. The lightning is mapped in North-South vs. East-West. The positive leaders on the left hand side is mapped by needle like structures occurring along main channel labeled N1-N11. While the negative leader on the right hand side come solely from the leader main channel. 


\section{High Energy Atmospheric physics}

TGFs are bursts of gamma rays produced in our atmosphere. Some of the top ten questions in lightning research at the time of writing of this proceeding are: what is the mechanism responsible for producing TGFs? How are intra-cloud discharges related to TGFs that are mainly observed by orbiting satellites. Can TGFs observed from the ground help us to a better understanding of the mechanisms responsible for producing TGFs?

The main runaway electron production mechanisms that are believed to be candidates for TGF production are: the Relativistic Runaway Electron Avalanche (RREA) [Gurevich, 1961] and the relativistic feedback mechanism [Dwyer, 2003]. Those mechanisms require the injection of seed electrons supplied by either cosmic ray EAS particles or by electrons accelerated directly from the free thermal electron population [Dwyer and Uman, 2014]. These mechanisms are tuned to interpret the observations of the large fluxes and the spectrum measurements observed by satellite and ground measurements.

Satellite experiments have been the main source of TGF observations since 1994. They are a few hundreds of kilometers away from the the TGF sources $(600-700 \mathrm{~km})$. If we are able to observe TGFs from the ground ( a few kilometers away) than we have the advantage of being much closer to the source which would allow us to collect further details about the TGFs initiation and development.

A few ground TGFs have been observed. The main challenges faced by ground TGF experiments is the strong attenuation of gamma rays by the atmosphere and the fact that the TGF detectors are low in altitude and not large enough to observe the full footprint of the TGF from the ground.

The Telescope Array detector, the largest Ultra High Energy Cosmic Rays (UHECR) detector in the Northern hemisphere, not only addresses these issues but, together with a suite of lightning instruments including the LMA and INTF, has recently reported on major breakthroughs in TGF detection. TA is now the world's leading detector in observing downward terrestrial gamma rays. It reported for the first time a direct correlation of downward gamma ray observations to intra-cloud discharge.

\subsection{Study of Terrestrial Gamma ray Flashes and their Origin with Telescope Array:}

Terrestrial gamma-ray flashes (TGFs) are bursts of gamma-rays initiated in the Earth's atmosphere. The first detection of TGFs was reported in 1994 by the Burst and Transient Source Experiment (BATSE) on the Compton Gamma-Ray Observatory satellite [Fishman et al., 1994, Kouveliotou, 1994]. Since then, several observations have shown that satellite-detected TGFs are associated with lightning flashes. In a normal polarity thunderstorm, a high-level intra-cloud lightning flash begins with a negative leader propagating upward from the mid-level negative charge region towards the upper positive charge region [Stanley et al., 2006, Shao et al., 2010, Lu et al., 2010, Cummer et al., 2015]. Recently TGFs observed by the Fermi Gamma-ray Burst Monitor (GBM) have been connected to energetic in-cloud pulses EIP [Lyu et al., 2016] . An EIP is known as a very energetic initial breakdown pulse with quite large peak currents of 150-300 kA.

Only a few TGFs have been observed from the ground before the Telescope Array TGF observations. Those naturally occurring TGFs have been observed to have gamma-rays after the return strokes stage of the flash, indicating that gamma-rays seen on the ground may originate from a 
mechanism different from that of the TGF satellite events [Dwyer et al., 2012, Tran et al., 2015, Dwyer et al., 2004, Hare et al., 2016] .

The TA detector is located in Millard County, Utah and is comprised of 507 scintillator detectors on a $1.2 \mathrm{~km}$ square grid covering $700 \mathrm{~km}^{2}$ Surface Detector (SD) array [Abu-Zayyad et al., 2013]. The Telescope Array Surface Detector (TASD), has an approximately 100\% duty cycle, and provides shower footprint information including core location, lateral density profile, and timing.

Between 2008-2013 ten bursts of consecutive TASD triggers were recorded in $1 \mathrm{~ms}$ time intervals. Since the TASD mean trigger rate is less than $0.01 \mathrm{~Hz}$, it is extremely unlikely that such a burst could be caused by accidental coincidence of high-energy cosmic rays. These bursts showed a strong correlation between bursts of energetic particle showers and NLDN lightning activity [Abbasi et al., 2017a].

In 2013 nine Lightning Mapping Arrays (LMA) were deployed throughout the Telescope Array site. The Langmuir LMA utilizes low-VHF (60-66 MHz) radio emissions in order to create 3-dimensional reconstruction of a lightning flash.

During 2014-2015, three flashes were observed in correlation with LMA, shown in Figure 5 [Abbasi et al., 2017b]. Figure 5 shows the TASD and the NLDN trigger time relative to the LMA source heights versus time. In each case the TASD triggers (dashed red lines) occurred within the first 1-2 ms of the flash, as the initial negative breakdown descended toward and into the lower positive charge region.

A typical trigger burst event waveform recorded by a SD together with the corresponding TASD footprint $(\sim 3-5 \mathrm{~km}$ in diameter) is shown in Figure 6. Each circle in the TASD shower footprint is a triggered SD with a color related to its relative arrival time. The size of the circle is proportional to the logarithm of the energy deposited in the SD. The number on each circle is the integrated area under the photomultiplier waveform VEM (one VEM corresponds to $\approx 2 \mathrm{MeV}$ ). The LMA and the the Vaisala NLDN source events are indicated by stars and diamonds respectively.

In each case radiation bursts are produced during downward negative breakdown at the beginning of low-altitude cloud-to-ground and intracloud flashes. The bursts occurred during the first $1-$ $2 \mathrm{~ms}$ of the discharges and had an overall duration between 87 and $551 \mu \mathrm{s}$. With the high-resolution timing of the TASD, the bursts are found to consist of several (2-5) individual components, each of which a few microseconds in duration, separated in time by $\simeq 10-250 \mu$ s between events. The bursts were also found to be forward beamed with footprints of $3-5 \mathrm{kms}$ in diameter.

The observed bursts are consistent with TGFs produced by the negative downward lightning leaders. This is the first ground observation of a downward TGF found to be an analog of the satellite observed TGFs produced by upward negative breakdown at the beginning of intracloud discharges. Note that all previous ground based TGF detections have reported observations of gamma-rays after the return strokes, which implies a different mechanism to originate ground based TGFs [Dwyer, 2012, Tran et al., 2015].

This result suggests that TGFs were almost certainly produced by one or two particularly energetic leader steps at the beginning of the breakdown. From this, the TGFs were possibly produced by "initial breakdown pulses" (IBPs) at the beginning of IC and CG flashes. To investigate this correspondence, an interferometer, in addition to slow and fast electric field sensors, were deployed at the TASD detector, at the end of July 2018. This enables us to study the relation between SD observations and the development of negative breakdown in greater detail. 
The Telescope Array detector, an UHECR detector, with over twenty five downward observed TGFs since 2008, provides us with the worlds largest sample of ground based TGF observations. It also provides us with possibly the first direct insight into the origin of ground observed TGFs.

The TASD has been expanded by a factor of four in coverage area, in which the TGF and lightning observations are continuing. Future observations of TGFs will continue to shed the light on the mysteries of their production and propagation.
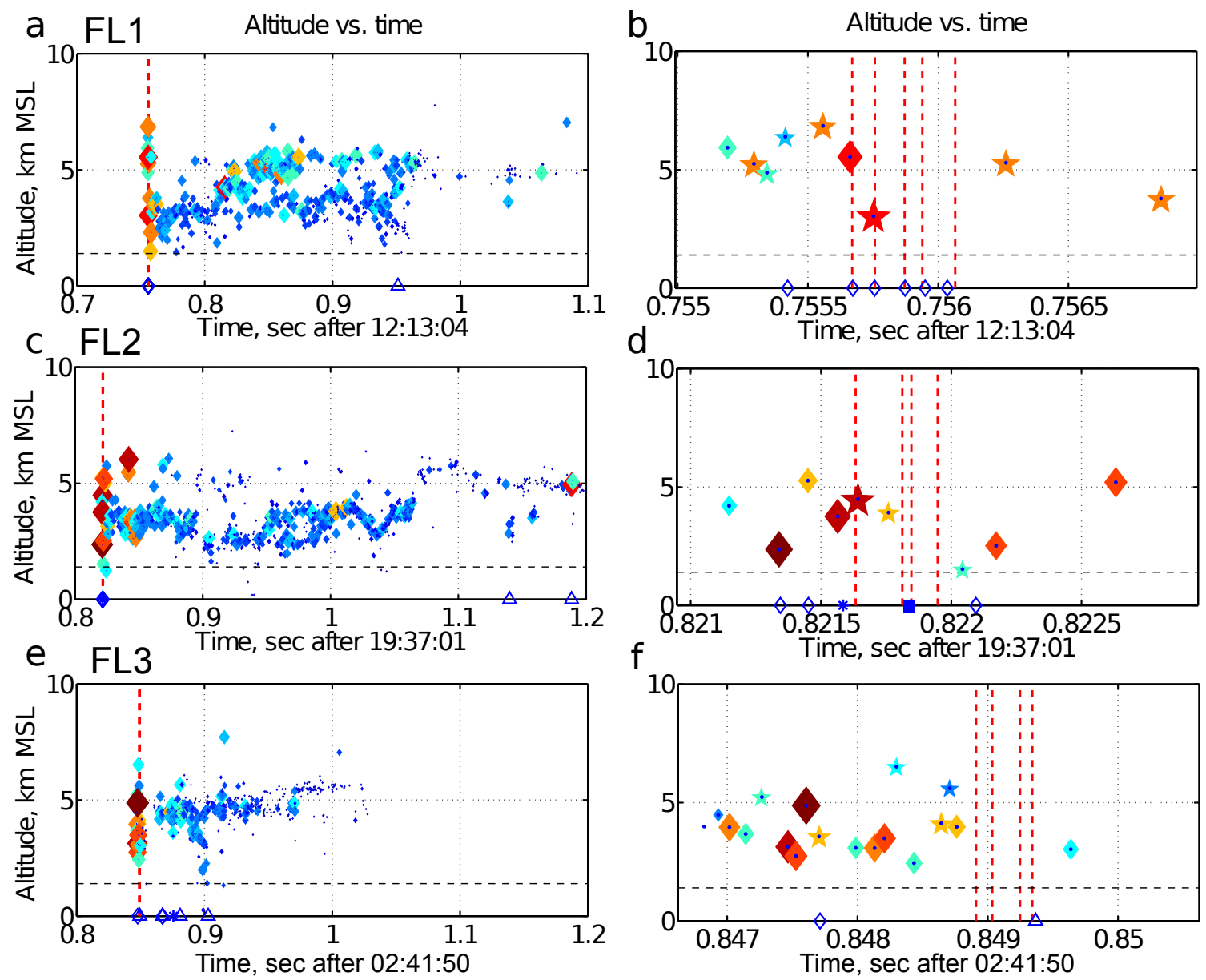

Figure 5: Observations of the LMA-correlated TASD bursts, showing altitude versus time plots of the LMA sources (colored diamonds) and the TASD trigger times (red dashed lines). The left panels show the complete flashes and the right panels show zoomed-in views during the first 2-3 ms of each flash. The LMA sources are colored and sized by the log of their radiated power, and range from source powers of $-20 \mathrm{dBW}$ (10 mW; blue colors) up to $+25 \mathrm{dBW}(320 \mathrm{~W}$; red colors). NLDN events are shown on the abscissa: $\diamond=$ $-\mathrm{IC}, \triangle=-\mathrm{CG}, \mathbf{\square}=+\mathrm{IC}, *=+\mathrm{CG}$. 

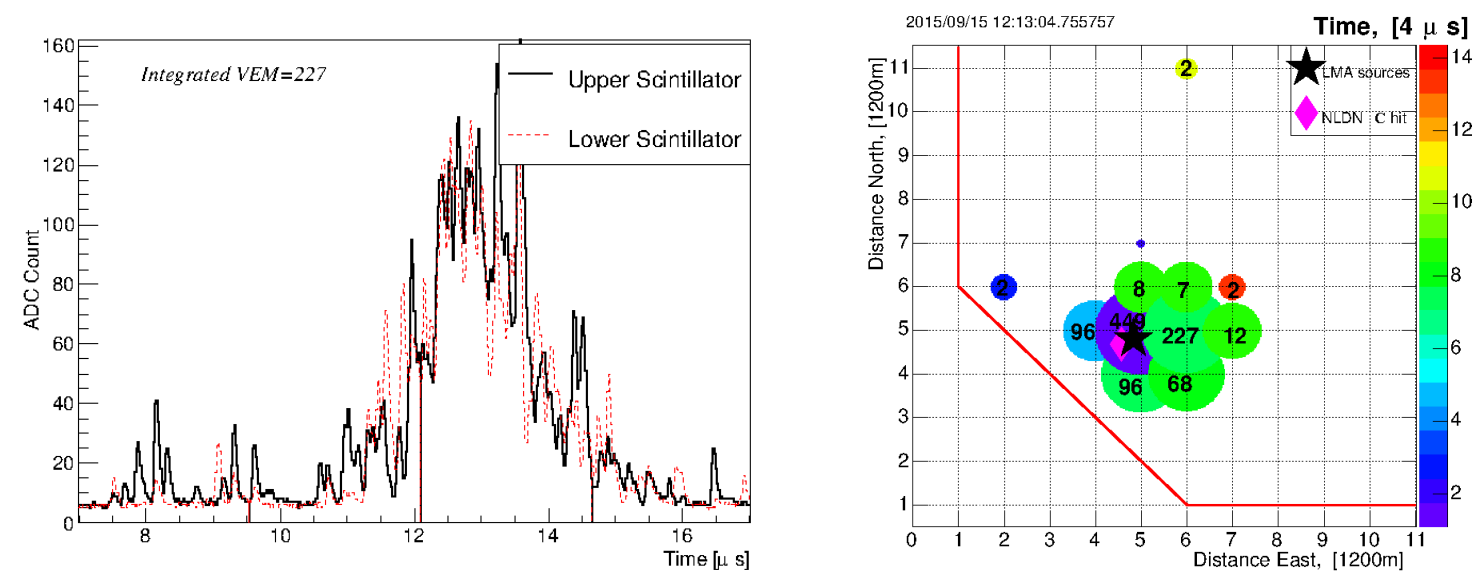

Figure 6: Left: Upper and lower scintillator waveforms in a single surface detector unit, for the second trigger in the LMA-correlated energetic radiation burst observed at 12:13:04 on 15 Sept. 2015. Right: Footprint of TASD hits for all detectors units involved in the sec- ond trigger of the burst, with the numbers indicating the Vertical Equivalent Muon (VEM) counts, and the color indicating the relative arrival times. Initial LMA and NLDN events are indicated by stars and diamonds respectively. The red line indicates the southwestern boundary of the TASD array. 

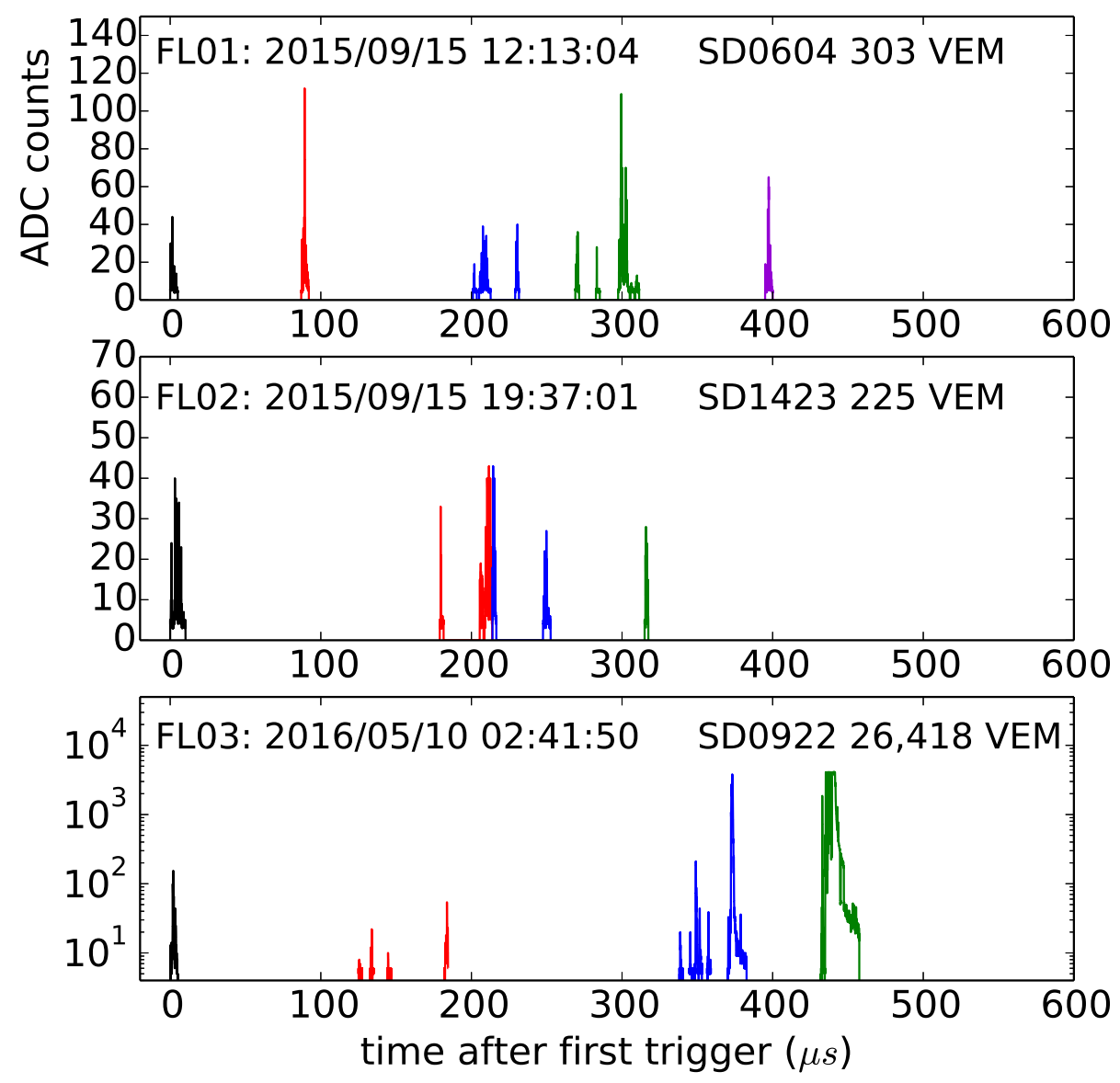

Figure 7: Top: the combined waveform, for one of the SD (XXYY=0604), for all five triggers observed in the burst FL01. Each trigger in the burst is colored individually. Triggers 1 through 5 are colored in black, red, blue, green and violet. Middle: same, except at SD (XXYY=1423) for the burst of four triggers observed in FL02. Bottom: same, except at SD (XXYY=0922), for the burst of four triggers observed in FL03. The waveforms are found to be temporally resolved into discrete components, most of which are less than 10 microseconds in duration and which occur in succession over a duration of a few hundred microseconds. 


\subsection{The First Observation of a GV Potential with G3MT:}

The GRAPES-3 Muon Telescope (G3MT) studies cosmic rays through the measurement of muon intensity. Located in Ooty in India $2200 \mathrm{~m}$ A.S.L. G3MT is composed of proportional counters (PRC) made from steel pipes. The PRCs are organized in a four layer configuration, under a thick $\sim 2 m$ concrete roof, covering $560 \mathrm{~m}^{2}$ in area. Such a configuration enables measuring muons with an energy threshold of $E_{\mu}=1 \sec (\theta) \mathrm{GeV}$ and an angular accuracy of $4^{\circ}$ [Hariharan et al., 2019].

G3MT scientists noticed that there exist rapid ( $\sim 10$ minutes) changes in the observed muon intensity related to thunderstorms. To better understand this relation, four Electric Field Mills (EFMs), were installed a few kilometers apart at GRAPES-3 [Hariharan et al., 2019].

The data collected by G3MT and the EFMs, between 2011-2014, confirmed the observation of 184 storms in coincidence. Seven of the most energetic thunderstorms, of these 184 events, reported a maximum muon intensity variation $\left(\Delta I_{\mu}\right)$ of greater than or equal to 0.4 percent.

The detector response to thunderstorms was studied using a uniform electric field, with an assumed cloud thickness, via simulation from CORSIKA code [Heck et al., 1998] (using Fluka [B Ãúhlen et al,, 2014] for low energy interactions $(E<80 \mathrm{GeV})$ and SYBILL [Ahn et al., 2009] for high energy interactions). It was noted from these simulations, that the observed $\Delta I_{\mu}$, is dependent on changes in the cloud potential. If the electric field and the cloud size was varied while keeping the potenital constant, $\Delta I_{\mu}$ would remain unchanged. This fact, together with the G3MT energy threshold of one $\mathrm{GeV}$ in energy, makes G3MT an ideal detector to measure high potential thunderstorms.

Only one of the seven most energetic thunderstorms were successfully modeled. The rest were too complicated to reproduce (possible complex E field structure). The modeled event was detected on the 1st of December 2014. It lasted for 18 minutes and produced a 20 sigma deficit in the observed muon intensity $\left(\Delta I_{\mu}\right)$ with a maximum of variation $-2 \%$, as shown in Figures 8 .

The dependence of $\Delta I_{\mu}$ on the potential $(\mathrm{V})$ is obtained from simulating a uniform electric field in a two kilometer thick cloud at an eight kilometers height A.S.L. The potential in the simulation was varied from -3 to $+3 \mathrm{GV}$ in steps on $0.1 \mathrm{GV}$. Figure 9 shows a positive potential (V), with a magnitude of approximately one $\mathrm{GV}$, would result in a $-2 \%$ variation in the maximum muon intensity.

Further characterization of the thunderstorm observed on December 2014 was attempted. While combining the angular velocity of $6.2^{\circ} / \mathrm{min}$ as observed by the EFMs and the E-W thunderstorm speed of $1 \mathrm{~km} / \mathrm{min}$ from the $\Delta I_{\mu}$ footprint one can calculate the height of the observed thunderstorm to be $11.4 \mathrm{~km}$ A.S.L. Doing the same for N-S the muons cover the full field of view provides a cloud size of about $11 \mathrm{~km}$, which yields to the calculation of the total area of this cloud to be $380 \mathrm{~km}^{2}$.

It is worth noting that such characterization of thunderstorm height and size further demonstrate the unique advantage of G3MT to study massive thunderstorm potential from the ground in comparison to balloon or aircraft experiments. The muon intensity change observed by G3MT enables us to describe the potential difference in these thunderstorms from the ground without dealing with all the limitation of narrow exposure in time and space using balloons and aircraft detectors. This allowed for the first observation of a one GV potential in thunderstorms, were the maximum potential observed by an air balloon experiment was reported to be $0.1 \mathrm{GV}$ [Marshall et al., 2001]. This could potentially explain the presence of hundreds of MV potentials TGFs observation [Hariharan et al., 2019]. 


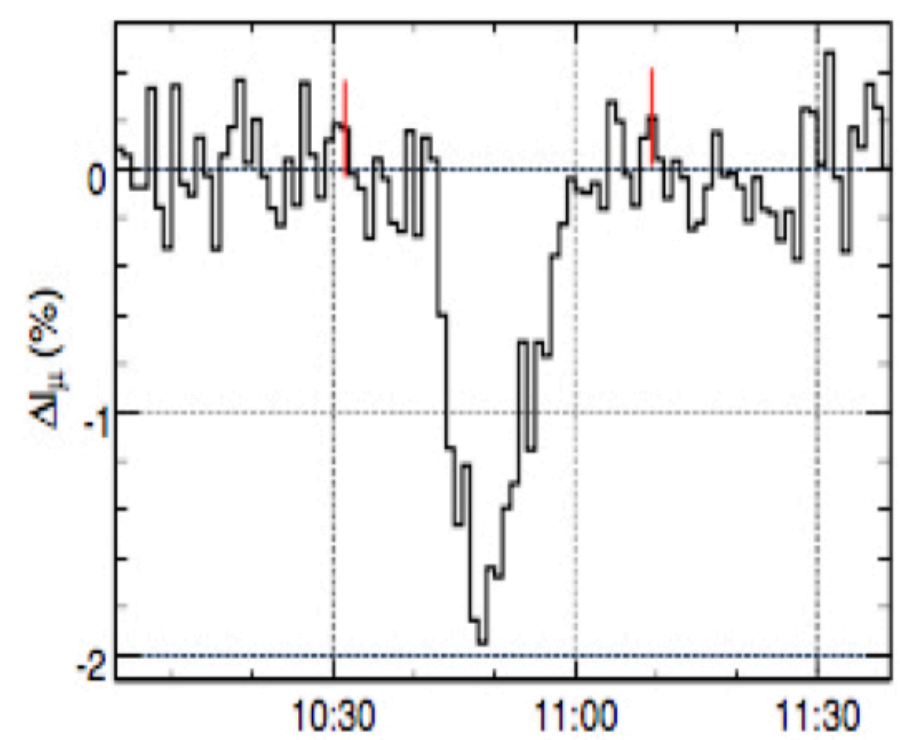

Figure 8: The maximum muon intensity variation $\left(\Delta I_{\mu}\right)$ vs. time. The intensity variation lasted 18 minutes at a maximum of $-2 \%$ variation. The vertical bar represents the $\pm 1 \sigma$ error.

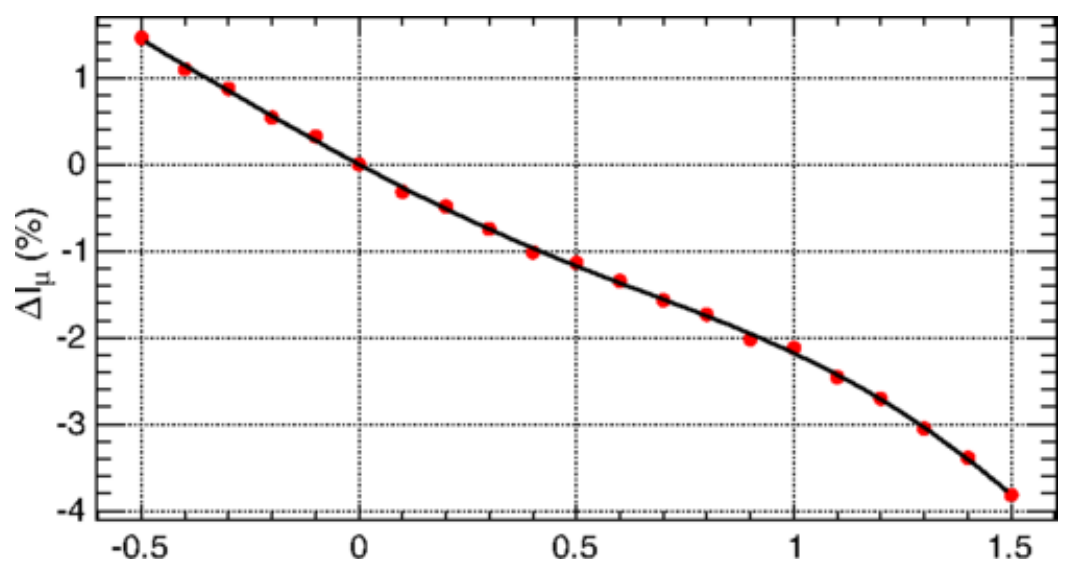

Figure 9: The muon intensity variation $\left(\Delta I_{\mu}\right)$ vs. the thunderstorm potential in GV. in steps of $0.1 \mathrm{GV}$. Note 


\section{Elves Detection and Classification:}

Elves are rapidly expanding optical emissions in the lower ionosphere. This believed to be induced by electromagnetic field pulses emitted by lightning. Elves were theoretically predicted by [Inan et al., 1991] and have been first observed from space by [Boeck et al., 1992]. They are now known to extend over several hundred kilometers at altitudes ranging from 70 to $95 \mathrm{~km}$ and have a duration of less than a millisecond.

The Auger Observatory [ABR, 2010] with it's 24 Fluorescence Detectors and their time resolution provide a unique opportunity to observe, study, and possibly model Elves. The first Elf was detected by Auger in 2005 after a dedicated more efficient trigger was implemented in 2013. Between 2013-2018 over 4000 Elves were observed by the Auger observatory.

Figure 10 shows two events displays by the Auger FDs together with the ADC traces of some of these triggered pixels. The ADC trace are observed to be either in an asymmetric gaussian or in more than one pulse in the traces. The later ones are referred to as multiple elves.

Multiple elves are categorized into four different categories based on the separation of the pulses in the pixels $(\Delta T)$ vs. the time of the first pulse in the multiple elves $\left(T_{i}\right)$, as shown in Figure 11. The four different categories are summarized as follows:

- $\Delta T<50 \mu s$. These events are explained by the possibility of the reflection of the EMP from intracloud lightning bouncing off of the ground.

- $\Delta T$ is larger, i.e. $\approx 120 \mu \mathrm{s}$, as shown in Figure 11 . These events are thought to be possibly from the IBP or the return stroke part of lightning.

- $\mathrm{n} \Delta T$ vs. $T_{i}$ is linearly decreasing. This could possibly be interpreted due to the interference of a signal from a single elve together with another class of light transient.

- $\Delta T$ abruptly changes. These observations have been suggested to be connected to TGFs.

Continuous efforts are ongoing to understand and model the Elves observed by the Auger FD detector. 

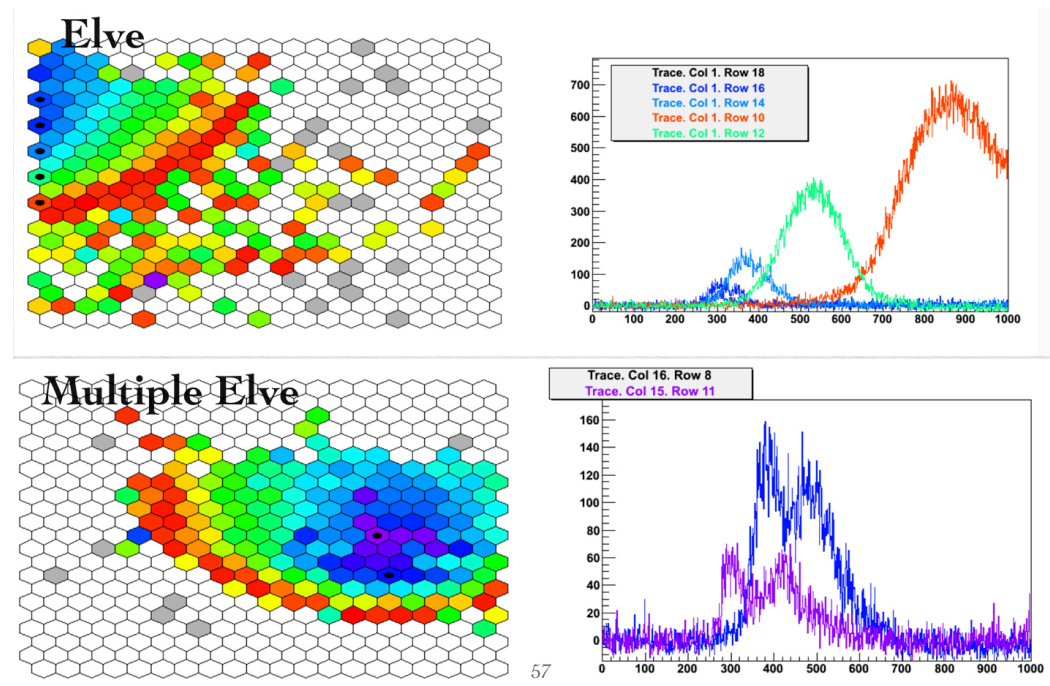

Figure 10: The left hand side plots are Elf and multiple Elf as observed on the telescope camera. The color scale is time. Blue is earlier red is later. The plots on the right are the ADC traces for the pixels marked in black points in the plots on the L.H.S. The ADC trace is an asymmetric gaussian for elves and contains several peaks in over ten pixels for events that are categorized as multiple elves. Note that the time bins are in 100 nano seconds bins.

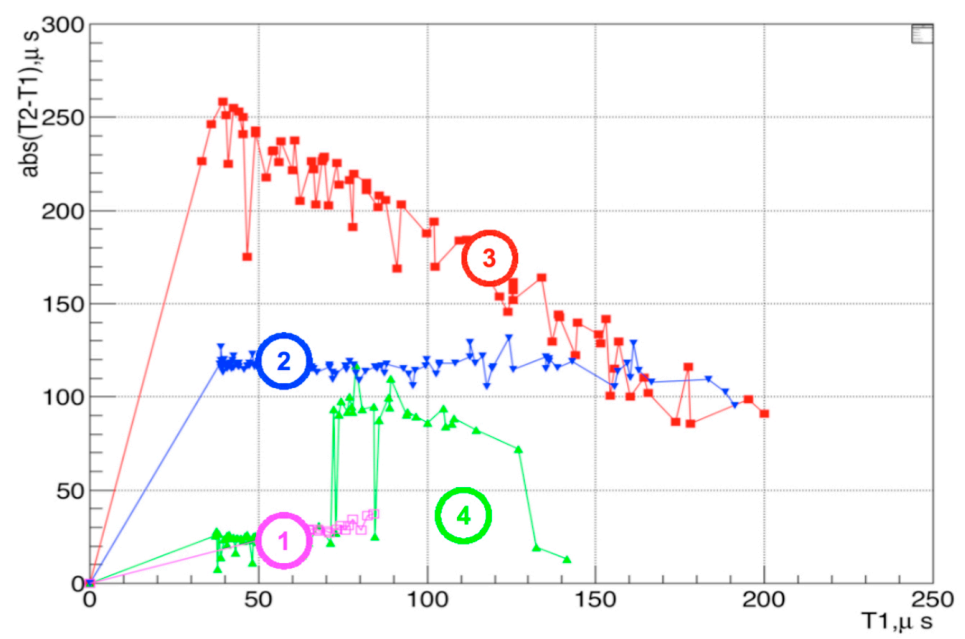

Figure 11: The difference in time between two pulses vs. time of the first pulse in multiple Elves as observed by Auger. This figure illustrates the summery of the multiple elves categories. 


\section{Summary and Conclusion}

We are living in an exciting time were several important observations by ground based cosmic ray experiments are contributing, in a unique way, to solving decades of mysteries related to lightning initiation, lightning propagation, and the origin of Terrestrial Gamma-ray Flashes. These ground cosmic ray experiment were not built to study atmospheric electricity but found themselves contributing to atmospheric physics significantly:

- LOFAR, with an unprecedented precision in mapping lightning which allows us to understand needles and their propagation.

- The Telescope Array, with the world largest sample of downward TGFs, in addition to breakthroughs in the origin of the source of TGFs.

- G3MT, with the first direct evidence of one GV potential inside thunderstorms.

- AUGER, with thousands of TLEs observations, that could possibly advances our understanding of their production and propagation.

\section{Acknowledgment:}

Thanks to Prof. John Belz for conversations and edits for this proceeding. Thanks to Prof. Pierre Sokolsky for his advice and help with editing this proceeding.

\section{References}

[ABR, 2010] (2010). The fluorescence detector of the pierre auger observatory. Nuclear Instruments and Methods in Physics Research Section A: Accelerators, Spectrometers, Detectors and Associated Equipment, 620(2):227 - 251.

[Abbasi et al., 2017a] Abbasi, R. et al. (2017a). The bursts of high energy events observed by the telescope array surface detector. Physics Letters A, 381(32):2565 - 2572.

[Abbasi et al., 2017b] Abbasi, R. U. et al. (2017b). Gamma-ray Showers Observed at Ground Level in Coincidence With Downward Lightning Leaders. [J. Geophys. Res. Atmos.123,6864(2018)].

[Abu-Zayyad et al., 2013] Abu-Zayyad, T. et al. (2013). The surface detector array of the Telescope Array experiment. Nucl. Instrum. Meth., A689:87-97.

[Ahn et al., 2009] Ahn, E.-J., Engel, R., Gaisser, T. K., Lipari, P., and Stanev, T. (2009). Cosmic ray interaction event generator sibyll 2.1. Phys. Rev. D, 80:094003.

[Boeck et al., 1992] Boeck, W. L. et al. (1992). Lightning induced brightening in the airglow layer. Geophysical Research Letters, 19(2):99-102.

[BÃúhlen et al., 2014] BÃúhlen, T. et al. (2014). The fluka code: Developments and challenges for high energy and medical applications. Nuclear Data Sheets, 120:211 - 214.

[Cummer et al., 2015] Cummer, S. A. et al. (2015). Lightning leader altitude progression in terrestrial gamma-ray flashes. Geophys. Res. Lett., 42:7792âĂŞ7798. 
[Dwyer et al., 2004] Dwyer et al. (2004). A ground level gamma-ray burst observed in association with rocket-triggered lightning. Geophysical Research Letters, 31(5). L05119.

[Dwyer et al., 2012] Dwyer et al. (2012). Observation of a gamma-ray flash at ground level in association with a cloud-to-ground lightning return stroke. Journal of Geophysical Research: Space Physics, 117(A10). A10303.

[Dwyer, 2003] Dwyer, J. R. (2003). A fundamental limit on electric fields in air. Geophysical Research Letters, 30(20).

[Dwyer, 2012] Dwyer, J. R. (2012). The relativistic feedback discharge model of terrestrial gamma ray fla shes. J. Geophys. Res., 117:A02308.

[Dwyer and Uman, 2014] Dwyer, J. R. and Uman, M. A. (2014). The physics of lightning. Physics Reports, 534(4):147 - 241. The Physics of Lightning.

[Fishman et al., 1994] Fishman, G. J. et al. (1994). Discovery of intense gamma-ray flashes of atmospheric origin. Science, 264(5163):1313-1316.

[Fitzgerald, 1984] Fitzgerald, D. R. (1984). Electric field structure of large thunderstorm complexes in the vicinity of cape canaveral. pages 260-262.

[Gurevich, 1961] Gurevich, A. (1961). On the theory of runaway electrons. Soviet Phys. JETP, 12(5):904âĂŞ912.

[Hare et al., 2016] Hare, B. M. et al. (2016). Ground-level observation of a terrestrial gamma ray flash initiated by triggered lightning. J. Geophys. Res., 121:6511-6533.

[Hare et al., 2018] Hare, B. M. et al. (2018). Lofar lightning imaging: Mapping lightning with nanosecond precision. Journal of Geophysical Research: Atmospheres, 123(5):2861-2876.

[Hare, 2019] Hare, B. M. o. (2019). Needle-like structures discovered on positively charged lightning branches. Nature, 568:360-363.

[Hariharan et al., 2019] Hariharan, B. et al. (2019). Measurement of the electrical properties of a thundercloud through muon imaging by the grapes-3 experiment. Phys. Rev. Lett., 122:105101.

[Heck et al., 1998] Heck, D., Knapp, J., Capdevielle, J. N., Schatz, G., and Thouw, T. (1998). CORSIKA: A Monte Carlo code to simulate extensive air showers.

[Huege et al., 2013] Huege, T., Ludwig, M., and James, C. W. (2013). Simulating radio emission from air showers with CoREAS. AIP Conf. Proc., 1535(1):128.

[Inan et al., 1991] Inan, U. S. et al. (1991). Heating and ionization of the lower ionosphere by lightning. Geophysical Research Letters, 18(4):705-708.

[Kouveliotou, 1994] Kouveliotou, C. (1994). BATSE results on observational properties of gamma-ray bursts. The Astrophysical Journal Supplement Series, 92:637-642.

[Lu et al., 2010] Lu, G. et al. (2010). Lightning mapping observation of a terrestrial gamma ray flash. Geophys. Res. Lett., 37:L11806.

[Lyu et al., 2016] Lyu, F. et al. (2016). Ground detection of terrestrial gamma ray flashes from distant radio signals. Geophysical Research Letters, 43(16):8728-8734.

[Marshall et al., 2001] Marshall, T. C. et al. (2001). Voltages inside and just above thunderstorms. Journal of Geophysical Research: Atmospheres, 106(D5):4757-4768. 
[Nelles et al., 2015] Nelles, A., Buitink, S., Falcke, H., HÃuurandel, J. R., Huege, T., and Schellart, P. (2015). A parameterization for the radio emission of air showers as predicted by coreas simulations and applied to lofar measurements. Astroparticle Physics, 60:13 - 24.

[Rees, 1956] Rees, W. C. T. (1956). A theory of thundercloud electricity. pages $236-297$.

[Rison et al., 1999a] Rison, W. et al. (1999a). A gps-based three-dimensional lightning mapping system: Initial observations in central new mexico. Geophysical Research Letters, 26(23):3573-3576.

[Rison et al., 1999b] Rison, W. et al. (1999b). A gps-based three-dimensional lightning mapping system: Initial observations in central new mexico. Geophysical Research Letters, 26(23):3573-3576.

[Schellart et al., 2015a] Schellart, P. et al. (2015a). Probing atmospheric electric fields in thunderstorms through radio emission from cosmic-ray-induced air showers. Phys. Rev. Lett., 114:165001.

[Schellart et al., 2015b] Schellart, P. et al. (2015b). Probing Atmospheric Electric Fields in Thunderstorms through Radio Emission from Cosmic-Ray-Induced Air Showers. Phys. Rev. Lett., 114(16):165001.

[Shao et al., 2010] Shao, X. et al. (2010). A closer examination of terrestrial gamma ray flash related lightning processes. J. Geophys. Res., 115:A00E30.

[Stanley et al., 2006] Stanley et al. (2006). A link between terrestrial gamma-ray flashes and intracloud lightning discharges. Geophysical Research Letters, 33(6). L06803.

[Stock et al., 2014] Stock, M. G. et al. (2014). Continuous broadband digital interferometry of lightning using a generalized cross-correlation algorithm. Journal of Geophysical Research: Atmospheres, 119(6):3134-3165.

[Stolzenburg et al., 2007a] Stolzenburg, M. et al. (2007a). Electric field values observed near lightning flash initiations. Geophysical Research Letters, 34(4).

[Stolzenburg et al., 2007b] Stolzenburg, M. et al. (2007b). Electric field values observed near lightning flash initiations. Geophysical Research Letters, 34(4).

[Tran et al., 2015] Tran, M. et al. (2015). A terrestrial gamma-ray flash recorded at the lightning observatory in gainesville, florida. Journal of Atmospheric and Solar-Terrestrial Physics, 136, Part A:86 - 93. Advances in Lightning Research.

[Weber et al., 1982] Weber, M. et al. (1982). A thundercloud electric field sounding: charge distribution and lightning ( langmuir laboratory). Journal of Geophysical Research, 87(C9):7158-7169.

[Winchen et al., 2019] Winchen, T. et al. (2019). Cosmic Ray Physics with the LOFAR Radio Telescope. J. Phys. Conf. Ser., 1181(1):012020.

[Winn and other, 1974] Winn, W. P. and other (1974). Measurements of electric fields in thunderclouds. Journal of Geophysical Research (1896-1977), 79(12):1761-1767. 